\title{
Organización retórica de la movida conclusión en ensayos de filosofía*
}

\author{
LORENA ECHEVERRÍA SÁNCHEZ*
}

Forma de citar este artículo: Echeverría, L. (2021). Organización retórica de la movida conclusión en ensayos de filosofía. Cuadernos de Lingüística Hispánica, (37), e12252.

Jol https://doi.org/ 10.19053/0121053X.n37.2021.12252

* Artículo de investigación.

** Magíster en Lingüística Aplicada de la Universidad Católica de la Santísima Concepción, Concepción, Chile. Académica en la Facultad de Estudios Teológicos y Filosofía de la Universidad Católica de la Santísima Concepción, Concepción, Chile. Proyecto Fondecyt 1170623. lecheverria@ucsc.cl @https://orcid. org/10.19053/0121053X.n37.2021.12194 


\section{Resumen}

El género argumentativo ensayo tiene amplia presencia en el contexto profesional de la filosofía, como un género relevante de su escritura académica. Promueve la comunicación, la reflexión crítica y habilidades argumentativas (Rodríguez, 2007; Oller, 2013, Rayas \& Méndez, 2017). Un apartado textual relevante para el cumplimiento del propósito comunicativo del género ensayo es la "conclusión". Existen estudios acerca de la organización retórica de las conclusiones en diversos géneros especializados (Ciapuscio \& Otañi, 2002; Espejo, 2006; Stagnaro, 2012; Fuentes, 2013), sin embargo, no destacan en la literatura investigaciones referentes a la organización retórica de la conclusión en ensayos de filosofía. Desde un paradigma comprensivo-interpretativo con un enfoque cualitativo de alcance exploratorio-descriptivo y con base en el modelo de análisis del género de Swales (1990, 2004), se realizó una descripción de la organización retórica que caracteriza la movida conclusión de un corpus de 69 ensayos de filosofía. Se destacan los pasos retóricos: identificación de la sección, síntesis final, replanteamiento de ideas e incorporación de nuevos argumentos. Estos pasos retóricos siguen una organización común en las conclusiones de ensayos de filosofía y el patrón más frecuente es la identificación de la sección seguida de la síntesis final, que permite reforzar la tesis del autor.

Palabras clave: ensayo, filosofía, organización retórica, conclusión.

\section{Rhetorical Organization of the Moving Conclusion in Philosophy Essays}

\section{Abstract}

The argumentative essay genre has a wide presence in the professional context of philosophy, as a relevant genre of its academic writing. It promotes communication, critical reflection, and argumentative skills (Rodríguez, 2007; Oller, 2013, Rayas \& Méndez, 2017). The "conclusion" is a relevant textual section for the fulfillment of the communicative purpose of the essay genre. There are studies about rhetorical organization of the conclusions in various specialized genres (Ciapuscio \& Otañi, 2002; Espejo, 2006; Stagnaro, 2012; Fuentes, 2013), however, research on rhetorical organization of the conclusion in philosophy essays does not stand up in literature. From a comprehensive-interpretive paradigm with a qualitative approach of exploratory-descriptive scope and based on the gender analysis model of Swales (1990, 2004), a description of the rhetorical organization that characterizes the moving conclusion of a corpus of 69 essays on philosophy was made. Rhetorical steps are highlighted: section identification, final synthesis, reformulation of ideas, and incorporation of new arguments. These rhetorical steps follow a common organization in the conclusions of philosophy essays, and the most frequent pattern is the identification followed by the final synthesis, which allows to reinforce the author's thesis.

Keywords: essay, philosophy, rhetorical organization, conclusion. 


\section{Organisation rhétorique de la conclusion mouvante dans les essais de philosophie}

\section{Résumé}

Le genre de l'essai argumentatif a une large présence dans le contexte professionnel de la philosophie, en tant que genre pertinent de son écriture académique. Il favorise la communication, la réflexion critique et les compétences argumentatives (Rodríguez, 2007; Oller, 2013, Rayas \& Méndez, 2017). Une section textuelle pertinente pour la réalisation de l'objectif de communication du genre d'essai est la «conclusion». Il existe des études sur l'organisation rhétorique des conclusions dans divers genres spécialisés (Ciapuscio \& Otañi, 2002; Espejo, 2006; Stagnaro, 2012; Fuentes, 2013). Néanmoins, elles ne mettent en relief dans la théorie les recherches concernant l'organisation rhétorique de la conclusion dans les essais de philosophie. A partir d'un paradigme interprétatif global avec une approche qualitative de portée exploratoire-descriptive et basée sur le modèle d'analyse de genre Swales (1990, 2004), on a achevé une description de l'organisation rhétorique qui caractérise la conclusion émouvante d'un corpus de 69 essais sur la philosophie. Les étapes rhétoriques sont mises en évidence: identification de la section, synthèse finale, reconsidération des idées et incorporation de nouveaux arguments. Ces étapes rhétoriques suivent une organisation commune dans les conclusions des essais de philosophie et le schéma le plus fréquent est l'identification de la section suivie de la synthèse finale, ce qui permet de renforcer la thèse de l'auteur.

Mots-clés: essai, philosophie, organisation rhétorique, conclusion.

\section{Organização retórica da movida conclusão em ensaios de filosofia}

\section{Resumo}

0 gênero ensaio argumentativo tem ampla presença no contexto profissional da filosofia, como gênero relevante de sua escrita acadêmica. Promove a comunicação, a reflexão crítica e a capacidade argumentativa (Rodríguez, 2007; Oller, 2013, Rayas \& Méndez, 2017). Uma seção textual relevante para 0 cumprimento do propósito comunicativo do gênero ensaio é a "conclusão". Existem estudos sobre a organização retórica das conclusões em vários gêneros especializados (Ciapuscio \& Otañi, 2002; Espejo, 2006; Stagnaro, 2012; Fuentes, 2013), mas as pesquisas sobre a organização retórica da conclusão em ensaios de filosofia não se destacam na literatura. A partir de um paradigma compreensivointerpretativo com abordagem qualitativa de âmbito exploratório-descritivo e com base no modelo de análise de gênero de Swales (1990, 2004), uma descrição da organização retórica que caracteriza a conclusão comovente de um corpus de 69 ensaios de filosofia. Destacam-se as etapas retóricas: identificação da seção, síntese final, repensar de ideias e incorporação de novos argumentos. Esses passos retóricos seguem uma organização comum nas conclusões dos ensaios de filosofia e o padrão mais frequente é a identificação da seção seguida da síntese final, o que permite reforçar a tese do autor.

Palavras-chave: ensaio, filosofia, organização retórica, conclusão 


\section{Introducción}

El análisis del discurso permite comprender las prácticas discursivas en las situaciones sociales en que se usa la lengua oral o escrita (Calsamiglia \& Tusón, 1999). Las comunidades discursivas hacen uso particular de la lengua en eventos comunicativos que les son propios y que asumen la forma de géneros discursivos especializados, cuyas características se han constituido en interesantes objetos de estudio para distintas disciplinas. De hecho, se han estudiado géneros discursivos propios de diversas áreas del saber, tales como economía (Stagnaro, 2012, 2015; Boudon \& Parodi, 2014; Parodi, 2015; González \& Burdiles, 2018); ciencias de la salud (Juárez, 2008; Olivares \& González, 2014; Burdiles 2016); ciencias biológicas (Ibáñez \& Moncada, 2017; García-Ramírez et al., 2017 ), por mencionar algunas. Sin embargo, géneros de áreas como la filosofía han sido escasamente estudiados, aun cuando entre ellos destacan el artículo de investigación, la lección, las obras completas, el tratado y el ensayo (Julio \& Parodi, 2017).

Las peculiaridades del ensayo son llamativas; no obstante, pocas investigaciones lo han estudiado como género discursivo especializado, lo que en el caso de la filosofía parece necesario examinar, dado que, en esta comunidad discursiva, la producción y sobre todo la publicación de escritos académicos como artículos de investigación y ensayos se consideran indispensables (0ller, 2013), lo que también demuestran las publicaciones de estos en la actualidad.

Por medio del ensayo, el autor busca poner a prueba sus pensamientos y dejar constancia de su reflexión, aportando ideas sobre cómo se debería percibir su postura respecto a un tema en un área de conocimiento que domina, intentando resolver 0 plantear un problema, discutir o simplemente reflexionar sobre algo (Schara, 1994; Arenas, 1997; Jonassen \& Bosung, 2010). Dado que el propósito comunicativo del ensayo es convencer a sus posibles lectores acerca de una postura, en este género predomina la modalidad argumentativa, cuya organización básica está dada por la secuencia: introducción, desarrollo y conclusión (Sánchez, 2001; Rodríguez, 2007; Zunino \& Muraca, 2012; Angulo, 2013; Oller, 2013). Para lograr dicho propósito, un género discursivo emplea la lengua de un modo particular y asume una forma de organización retórica específica (Swales, 1990, 2004). En el caso del ensayo, tendrá suma relevancia el área en que se esté desarrollando, ya que el proceso mismo de escritura es un "proceso lingüístico-cognitivo y social llevado a cabo por un sujeto a partir de ciertos fines e inmerso en un determinado contexto del que es partícipe" (Farlora, 2015, p. 263). En filosofía, al igual que en otras disciplinas, el ensayo se considera un producto relevante de escritura académica, ya que promueve la comunicación y la reflexión crítica (Figueroa \& Aillon, 2015), y contribuye al desarrollo de habilidades argumentativas (Rayas \& Méndez, 2017). Estas habilidades argumentativas se harán efectivas en la formulación del pensamiento propio en el ensayo, lo cual conlleva tener en cuenta el propósito comunicativo que guiará las elecciones en el proceso de escritura y establecer pasos retóricos que configuren 
una organización retórica cuyos propósitos comunicativos serán coherentes con el propósito del género (Swales, 2004).

En relación con la movida conclusión, Swales (1990), al analizar la organización retórica del artículo de investigación, no hace una clara distinción entre discusión y conclusión, con lo cual se entiende que ambas se dan en conjunto y que la conclusión es parte de la discusión. Tomando en cuenta lo anterior, se puede presumir que, para identificar la movida conclusión en un texto, donde no se da de forma explícita, se pueden reconocer los pasos retóricos relacionados con los propósitos comunicativos propios del texto y, específicamente, del cierre discursivo. Dichos propósitos comunicativos, según Zunino y Muraca (2012) y Oller (2013), apuntan a resumir y evaluar resultados obtenidos, persuadir, disuadir y, en algunos casos, sugerir nuevas problematizaciones. Por su parte, Partarrieu (2018) indica que en las conclusiones de ensayos de filosofía escritos por estudiantes destacan los pasos síntesis final, reiteración de tesis, cita de otra fuente, valoración del tema o tesis, incorporación al lector, cuestionamientos e incorporación de nuevos argumentos. Estos pasos retóricos son los que permitirán la identificación de la movida conclusión en ensayos de filosofía.

Se ha indagado acerca de la organización retórica de las conclusiones en diversos estudios sobre géneros especializados, los cuales dan pistas de cómo identificar la movida conclusión (Hopkins \& Dudley-Evans, 1988; Ciapuscio \& Otañi, 2002; Espejo, 2006; Sabaj et al., 2011; Stagnaro, 2012; Fuentes, 2013; Soler-Monreal, 2016). Sin embargo, no destacan en la literatura investigaciones referentes a cómo se organizan los pasos retóricos presentes en el cierre discursivo de un género predominantemente argumentativo como el ensayo de filosofía.

Esta investigación se centrará en la manera en que se organiza retóricamente la movida conclusión de ensayos de filosofía publicados en revistas especializadas. Puntualmente, se indagará en los pasos retóricos que caracterizan la movida final de los ensayos.

Las preguntas de investigación que guían este estudio son las siguientes: (1) ¿Cómo es la organización retórica de la movida conclusión del ensayo escrito por expertos del área de la filosofía? (2) ¿Cuáles son los pasos retóricos más frecuentes para concluir y sus respectivos propósitos comunicativos en ensayos de filosofía escrito por expertos?

Para responderlas, se propone como objetivo general describir la organización retórica que caracteriza la movida conclusión de ensayos de filosofía. Como objetivos específicos, se persigue: (1) determinar los pasos retóricos de la movida conclusión en un corpus de ensayos de filosofía escritos por expertos, y (2) determinar el propósito 
comunicativo de los pasos retóricos presentes en la movida conclusión de los ensayos de filosofía.

Dada la ausencia de investigaciones centradas en una descripción del ensayo de filosofía, se espera con la presente investigación ampliar el conocimiento acerca del género ensayo, en particular sobre un apartado específico: la conclusión; además de contribuir al desarrollo de estrategias argumentativas requeridas en el proceso de alfabetización de quienes utilizan el ensayo en contextos profesionales, como el ámbito de la filosofía.

\section{Metodología}

Esta investigación se aborda desde un paradigma comprensivo-interpretativo, al centrar su atención en el uso de ciertos pasos retóricos y las razones de su uso para lograr los propósitos comunicativos que le corresponden a la movida conclusión. Adicionalmente, se identifica con un enfoque cualitativo, ya que se realizó un análisis basado en la recolección y observación de datos (Hernández et al., 2010) con el fin de describir detalladamente la organización retórica de la movida conclusión del género discursivo ensayo de filosofía. Finalmente, el alcance de la investigación se reconoce como exploratorio descriptivo, pues se enfoca en un aspecto poco conocido del género ensayo de filosofía, a saber, los pasos retóricos presentes en la movida conclusión de dicho género.

Este estudio se desarrolló en tres fases principales: en primer lugar, la conformación del corpus de estudio; luego, el diseño de una matriz de análisis y, finalmente, el análisis completo del corpus.

\section{Conformación del corpus ENS-FIL -2019 (Corpus de Ensayos de} Filosofía, 2019)

Se creó el corpus ENS-FIL-2019, conformado por 69 ensayos de filosofía escritos en español. Los criterios de selección fueron los siguientes: ensayos escritos por profesionales de la disciplina y publicados en revistas especializadas. Las revistas seleccionadas debían tener indexación Scielo o Dialnet y haber sido publicadas en países hispanohablantes. De las revistas seleccionadas se escogieron aquellas que contenían una sección dedicada a ensayos o que, en su defecto, en la sección Misceláneos incorporaran ensayos. Finalmente, conformaron el corpus los ensayos publicados entre los años 2010 y primer semestre del 2019, recolectados en formato digital, descargados de sus páginas oficiales (Tabla 1). Si bien se hizo una revisión de diversas revistas de filosofía, muchas de ellas no incluyen ensayos en sus publicaciones, o los publican como artículos, razón por la que hubo ensayos que no pudieron considerarse en el corpus analizado. Fue un criterio de inclusión que, si los ensayos estaban en la sección Misceláneos, estos se identificaran como ensayos en su resumen. 
Tabla 1. Corpus ENS-FIL-2019: número de ensayos por revista

\begin{tabular}{|l|l|}
\hline Revista & Ensayos \\
\hline Revista de Filosofía (Universidad de Chile, Chile) & 10 \\
\hline Revista de Humanidades (Universidad de Valparaíso, Chile) & 2 \\
\hline Paralaje (Pontificia Universidad Católica de Valparaíso, Chile) & 25 \\
\hline $\begin{array}{l}\text { Universitas Philosophica (Pontificia Universidad Javeriana, } \\
\text { Bogotá, Colombia) }\end{array}$ & 14 \\
\hline $\begin{array}{l}\text { Estudios de Filosofía (Instituto de Filosofía de la Universidad } \\
\text { de Antioquía, Colombia) }\end{array}$ & 9 \\
\hline Daimon (Universidad de Murcia, España) & 6 \\
\hline Factótum (Asociación Cultural Factótum, España) & 3 \\
\hline Total & $\mathbf{6 9}$ \\
\hline
\end{tabular}

\section{Delimitación del apartado por analizar}

Con el fin de tener una aproximación a las características organizacionales de la movida conclusión, se realizó la lectura de un microcorpus de ensayos de filosofía, compuesto por 15 textos, y se logró un primer acercamiento a las características de los pasos retóricos de la movida conclusión de los ensayos de filosofía.

\section{Identificación de pasos retóricos}

A partir de la información recogida en la fase anterior, más la proveniente de la literatura especializada, recolectada de las investigaciones acerca de organización retórica de autores como Hopkins y Dudley-Evans (1988), Sabaj et al. (2011), Fuentes (2013), Soler-Monreal (2016) y Partarrieu (2018), se diseñó una plantilla de análisis, que presentaba 14 pasos retóricos con sus respectivos propósitos comunicativos.

\section{Validación plantilla de análisis}

Para la validación de la plantilla de análisis se consultaron tres jueces expertos, todos profesionales del área de la filosofía, con el grado de doctor. Los jueces fueron capacitados previamente acerca de qué son y cómo se identifican los pasos retóricos que conforman la organización retórica de un género discursivo y cuáles son sus propósitos comunicativos. Posteriormente, los tres jueces recibieron una carpeta que contenía instrucciones para el análisis. Junto con ello obtuvieron una matriz de pasos retóricos y propósitos comunicativos, con ejemplos provenientes del microcorpus, una grilla de análisis y dos ensayos seleccionados de cada revista del corpus, cuyas 
conclusiones debían ser analizadas según las instrucciones. Además, se incluyó como ejemplo la conclusión de un ensayo del corpus con los pasos retóricos identificados. Cabe señalar que los textos analizados por los jueces correspondieron a ensayos completos, si bien las instrucciones estaban enfocadas a las conclusiones, se requería la lectura de la totalidad del documento.

Una vez que los jueces entregaron su análisis, se comparó el grado de acuerdo entre los expertos y, en conformidad con esto, se realizaron cambios pertinentes en la plantilla de análisis, tales como modificaciones sobre los pasos retóricos o la definición de sus propósitos comunicativos, con el fin de hacer más claras las definiciones. Con estos resultados se creó el instrumento de análisis definitivo.

\section{Análisis del corpus}

Una vez definida la matriz de análisis, esta se aplicó a todo el corpus, se identificaron los pasos retóricos presentes, con el propósito de cuantificar la presencia de cada movida y observar la predominancia o ausencia de algunas de ellas.

El análisis del corpus se llevó a cabo siguiendo la propuesta de Swales (1990, 2004) para definir la organización retórica de un género. Este estudia los rasgos retórico-discursivos de textos que constituyen un género. Los rasgos retóricodiscursivos, a su vez, comparten criterios comunicativos que son propios de una comunidad discursiva, ya que ella perseguirá objetivos comunes, por lo que sus necesidades comunicativas tenderán a mantener las características de los textos propios de un género (Swales, 1990).

Los datos que resultaron de este análisis se registraron codificados en una planilla Excel y se llevaron a frecuencias relativas para determinar la predominancia de algunos pasos retóricos que conforman la organización retórica de la movida conclusión del género discursivo ensayo, así como también sus propósitos comunicativos.

\section{Resultados y discusión}

\section{Variedad de pasos retóricos y propósitos comunicativos de la movida conclusión en ensayos de filosofía}

Como se señaló en el apartado anterior, el corpus consta de 69 conclusiones de ensayos de filosofía escritos en español por expertos del área. Su estudio se basó en el modelo de análisis de género propuesto por Swales (1990) y estuvo guiado por un listado de pasos retóricos propios del apartado conclusión, provenientes principalmente de las propuestas de diversos autores (Hopkins \& Dudley-Evans, 1988; Sabaj, et al., 2011; Fuentes, 2013; Soler-Monreal, 2016; Partarrieu, 2018).

El análisis del corpus permitió identificar 14 pasos retóricos presentes en la movida conclusión de los ensayos de filosofía, los que se presentan en la Tabla 2 con sus respectivos propósitos comunicativos. 
Tabla 2. Variedad de pasos retóricos y propósitos comunicativos en las conclusiones de ensayos de filosofía

\begin{tabular}{|c|c|c|}
\hline 1. & Pasos retóricos & Propósito comunicativo \\
\hline MR1. & Identificación de la sección & Anunciar el apartado final del ensayo \\
\hline MR2. & Síntesis final & $\begin{array}{l}\text { Sintetizar interpretaciones y argumentos } \\
\text { para dar validez a la tesis central }\end{array}$ \\
\hline MR3. & Replanteamiento de ideas & $\begin{array}{l}\text { Reformular una idea antes mencionada de } \\
\text { otra manera }\end{array}$ \\
\hline MR4. & Replanteamiento de tesis & $\begin{array}{l}\text { Asegurar la coherencia entre tesis e ideas } \\
\text { de la conclusión }\end{array}$ \\
\hline MR5. & Cita de otra fuente & $\begin{array}{l}\text { Dar solidez a la tesis recurriendo en } \\
\text { diversas partes de la conclusión a voces } \\
\text { especializadas }\end{array}$ \\
\hline MR6. & Cita final & $\begin{array}{l}\text { Cerrar la idea central con una cita que dé } \\
\text { validez a la tesis }\end{array}$ \\
\hline MR7. & Valoración del tema de tesis & Posicionar el ensayo como relevante \\
\hline MR8. & Incorporación del lector & Asegurar la adhesión del lector a la tesis \\
\hline MR9. & Planteamiento de preguntas retrospectivas & $\begin{array}{l}\text { Motivar en el lector la reflexión a través de } \\
\text { preguntas relacionadas con el ensayo. No } \\
\text { necesariamente están respondidas en la } \\
\text { conclusión }\end{array}$ \\
\hline MR10. & Planteamiento de preguntas prospectivas & $\begin{array}{l}\text { Invitar al lector a la reflexión a través de } \\
\text { preguntas planteadas por el autor, } \\
\text { motivadas por lo trabajado en el ensayo. } \\
\text { No necesariamente están respondidas en } \\
\text { la conclusión }\end{array}$ \\
\hline MR11. & Incorporación de nuevos argumentos & Reforzar ideas de la tesis \\
\hline MR12. & Ejemplificación & $\begin{array}{l}\text { Aportar a la comprensión de ideas por } \\
\text { medio de evidencias }\end{array}$ \\
\hline MR13. & Proyección & Sugerir nuevas líneas de investigación \\
\hline MR14. & Mención de otras investigaciones/autores & $\begin{array}{l}\text { Respaldar lo planteado en el ensayo } \\
\text { recurriendo, sin hacer una cita, a voces } \\
\text { autorizadas }\end{array}$ \\
\hline
\end{tabular}

Frecuencia de pasos retóricos presentes en la movida conclusión de ensayos de filosofía

Establecida la variedad de los pasos retóricos y sus propósitos comunicativos, se presentan los resultados obtenidos en cuanto a la frecuencia de aparición de cada uno de ellos en el análisis del corpus . La Tabla 3 muestra la frecuencia de los pasos retóricos en la columna 1 y su porcentaje en la columna 2. 
Tabla 3. Frecuencia de pasos retóricos en conclusiones de ensayos de filosofía

\begin{tabular}{|c|c|r|}
\hline Movidas retóricas & Total & \multicolumn{1}{|c|}{$\%$} \\
\hline Identificación de la sección & 39 & $57 \%$ \\
\hline Síntesis final & 64 & $93 \%$ \\
\hline Replanteamiento de ideas & 36 & $52 \%$ \\
\hline Replanteamiento de tesis & 21 & $30 \%$ \\
\hline Cita de otra fuente & 27 & $39 \%$ \\
\hline Cita final & 16 & $23 \%$ \\
\hline Valoración del tema de tesis & 10 & $14 \%$ \\
\hline Incorporación del lector & 5 & $7 \%$ \\
\hline Planteamiento de preguntas retrospectivas & 7 & $10 \%$ \\
\hline Planteamiento de preguntas prospectivas & 8 & $12 \%$ \\
\hline Incorporación de nuevos argumentos & 41 & $59 \%$ \\
\hline Ejemplificación & 13 & $19 \%$ \\
\hline Proyección & 6 & $9 \%$ \\
\hline Mención de otras investigaciones & 27 & $39 \%$ \\
\hline
\end{tabular}

La Tabla 3, además de la variedad de 14 pasos retóricos que pueden manifestarse en la movida conclusión del ensayo de filosofía, expone la frecuencia con que cada uno de ellos aparece en el corpus. Se destaca un conjunto de cuatro pasos retóricos cuya frecuencia de aparición es superior al 50 \% (Figura 1) ; estos se determinan como las movidas retóricas prototípicas.

Figura 1. Movidas retóricas prototípicas

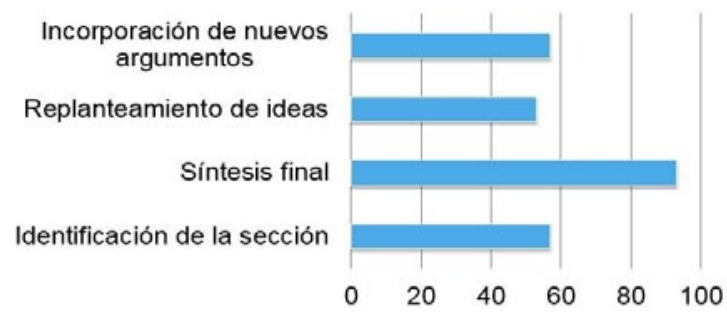

Los datos de la Tabla 3 permiten afirmar que los pasos síntesis final, incorporación de nuevos argumentos, identificación de la sección y replanteamiento de ideas son pasos prototípicos de las conclusiones de los ensayos de filosofía analizados. Esto quiere decir que, para cerrar discursivamente este tipo de ensayos en esta disciplina, es indispensable que se haga una síntesis de las interpretaciones y los argumentos desarrollados para dar validez a la tesis central; además, que se incorporen nuevos argumentos que la refuercen, que el apartado final del ensayo sea anunciado a partir de la identificación textual de la sección y, en menor grado, que se reformule una idea que haya sido mencionada anteriormente (Figura 1).

Estos resultados son coherentes con lo que plantean diversos autores (Sánchez, 2001; Rodríguez, 2007; Zunino \& Muraca, 2012; Angulo, 2013; Oller, 2013), en el 
sentido de que el propósito comunicativo del ensayo es convencer a sus posibles lectores acerca de una postura por medio de argumentos y síntesis de ideas, dada su modalidad argumentativa. Asimismo, estos hallazgos coinciden parcialmente con los resultados de Partarrieu (2018), en cuya investigación hizo un análisis comparativo de pasos retóricos en la movida conclusión de un conjunto de ensayos de filosofía escritos por estudiantes y de otro producido por expertos en el área. La autora observa en el corpus de expertos que la identificación de la sección y la síntesis son movidas peculiares de la conclusión en ensayos de filosofía.

Por otro lado, todos los pasos retóricos con frecuencia menor al $50 \%$ se identifican como movidas retóricas periféricas. Estos pasos periféricos pueden 0 no aparecer, puesto que su ausencia en las conclusiones no impedirá al apartado conclusión alcanzar su propósito comunicativo.

\section{Patrones de organización retórica en la conclusión en ensayos de filosofía}

La pregunta central de esta investigación busca responder cómo es la organización retórica de la movida conclusión en los ensayos de filosofía escritos por profesionales del área. Para intentar responder lo anterior, se hizo un registro de los patrones presentes en la movida conclusión del corpus analizado. Se entenderá por patrón a la "secuencia de segmentos textuales (orden y combinación)" (Ciapuscio \& Otañi, 2002, p. 119) formada por movidas retóricas.

En la Tabla 4 se observan los 8 patrones más comunes, presentados en cantidad y porcentaje de frecuencia de aparición.

Tabla 4. Patrones de organización retórica predominante en conclusión de ensayos de filosofía del corpus ENS-FIL-2019

\begin{tabular}{|c|c|c|}
\hline \multicolumn{3}{|c|}{$\begin{array}{l}\text { MR A: Identificación de la sección } \\
\text { MR B: Síntesis final } \\
\text { MR C: Incorporación de nuevos argumentos } \\
\text { MR D: Replanteamiento de ideas } \\
\text { MR E: Replanteamiento de tesis } \\
\text { MR F: Cita final }\end{array}$} \\
\hline Patrón & Cantidad & $\%$ Frecuencia relativa \\
\hline AEBC & 2 & $2 \%$ \\
\hline D & 3 & $4 \%$ \\
\hline $\mathrm{ABC}$ & 6 & $8 \%$ \\
\hline $\mathrm{AF}$ & 6 & $8 \%$ \\
\hline E & 9 & $12 \%$ \\
\hline $\mathrm{AD}$ & 16 & $23 \%$ \\
\hline B & 32 & $44 \%$ \\
\hline $\mathrm{AB}$ & 35 & $50 \%$ \\
\hline
\end{tabular}


En los 69 ensayos analizados se da una diversa organización de sus pasos retóricos, prácticamente diferente en cada ensayo, pero aun así se logra identificar la organización prototípica $\mathrm{AB}, \mathrm{B}$ y $\mathrm{AD}$. Un 50 \% de las conclusiones sigue el patrón $\mathrm{AB}$ (Ejemplo 1), en el que se identifica la sección conclusión con un subtítulo o marcador discursivo y se desarrolla una síntesis final del tema tratado en el ensayo. La repetición del patrón $\mathrm{AB}$ da cuenta de una funcionalidad de la parte textual (Ciapuscio \& Otañi, 2002), en la que el filósofo asume como importante sintetizar interpretaciones y argumentos para dar validez a la tesis central, lo que se reconoce como su propósito comunicativo.

Ejemplo 1. Ejemplo de patrón $\mathrm{AB}$ de la organización retórica de la movida conclusión en ensayos de filosofía

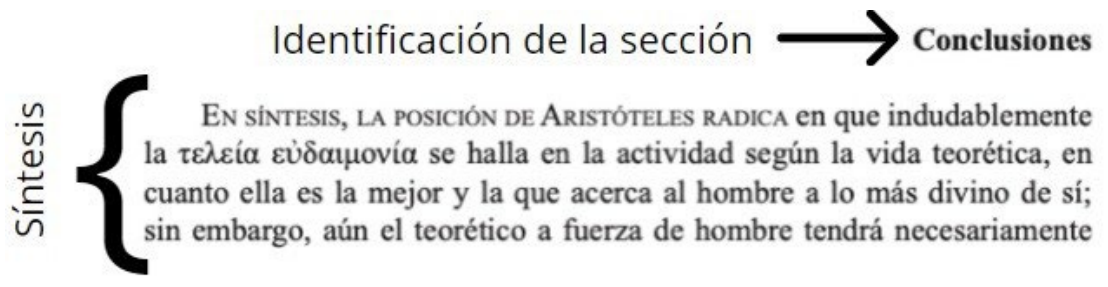

Por otra parte, un $44 \%$ de las conclusiones de los ensayos, que no están identificados por un subtítulo o marcador discursivo, solo desarrollan una síntesis final (Ejemplo 2). Esto deja entrever que la movida conclusión en un ensayo de filosofía se puede identificar por medio de la presencia de la síntesis, cuando la conclusión no se encuentre explícitamente marcada, es decir, por medio de un subtítulo. Finalmente, también se considera relevante el $23 \%$ correspondiente al patrón $\mathrm{AD}$ (Ejemplo 3) de la conclusión de los ensayos, que consiste en identificación de la sección y replanteamiento de ideas.

Ejemplo 2. Ejemplo de patrón B de la organización retórica de la movida conclusión en ensayos de filosofía

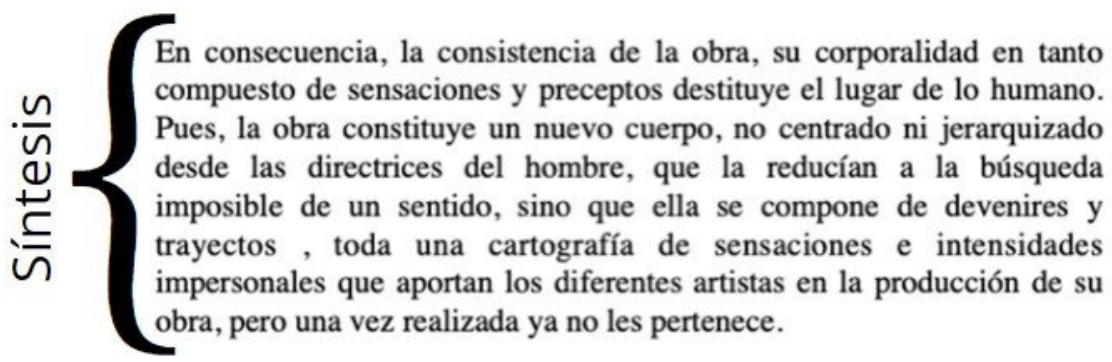


Ejemplo 3. Ejemplo de patrón $\mathrm{AD}$ de la organización retórica de la movida conclusión en ensayos de filosofía

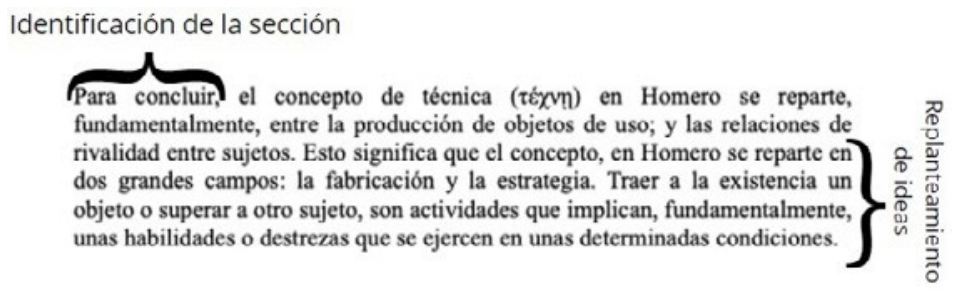

Reyes (1959) caracteriza el ensayo como el centauro de los géneros, pues considera difícil su clasificación por tener rasgos prototípicos difíciles de clasificar. En el análisis del presente estudio, centrado específicamente en ensayos de filosofía, se han observado variados pasos retóricos con sus respectivos propósitos comunicativos, los cuales a su vez se organizan de diversas formas a lo largo de la movida conclusión de los ensayos. Esto ha logrado identificar efectivamente rasgos prototípicos que caracterizarían el ensayo de esta disciplina en particular.

Siguiendo la misma línea de Cerezo (2002), se coincide en que el ensayo tiene una marca personal, una "constatación del yo" (Cerezo, 2002, p. 7) que variará dependiendo del propósito comunicativo de quien escribe, lo que se ve reflejado en el texto. Ahora bien, el análisis realizado a la movida conclusión de los 69 ensayos de filosofía que componen el corpus demuestra, por medio de sus rasgos prototípicos, que un propósito comunicativo central de la movida conclusión es sintetizar, incorporar nuevos argumentos y replantear ideas. Esta variación del propósito comunicativo dependerá del campo en que se desarrolle el ensayo; como plantean Rayas y Méndez (2017), la escritura del ensayo se materializará en torno a los objetivos propios de cada comunidad.

Se observa, además, el carácter argumentativo (Wingate, 2012) del ensayo de filosofía, porque dentro de sus rasgos prototípicos incorpora nuevos argumentos que, con una frecuencia del $59 \%$, buscan reforzar ideas de la tesis trabajada en el ensayo. También la frecuencia del replanteamiento de ideas (52\%) da cuenta del carácter reflexivo del ensayo para quien lo escribe, rasgo destacado por Angulo (2013, p. 109) al plantear que "el ensayo obliga a reflexionar" y el hecho de repetir una idea antes mencionada, de otra manera, requiere repensar lo ya expuesto para replantearlo y, por otro lado, motiva la comprensión del lector. Igualmente, se puede señalar que hay pasos retóricos no relevantes, como la incorporación del lector en la reflexión, que se encuentra escasamente identificado , como ocurre también en el estudio de Partarrieu (2018). Otros pasos retóricos periféricos son , a saber, incorporación del lector, proyección, preguntas retrospectivas, preguntas prospectivas y valoración del tema. Estos rasgos, siguiendo a Angulo (2013) y Arenas (1997), pueden aparecer porque en un ensayo se busca plasmar la postura personal del autor, quien pone a prueba sus pensamientos dejando constancia de su propia reflexión, pero no espera 
motivar la reflexión del lector. El autor solo busca aportar una idea que el lector entienda y a la cual se adhiera (Arenas, 1997) y, por ello, la conclusión solo se centra en reafirmar su postura frente al tema tratado.

Para lograr el objetivo principal, en este caso de la conclusión del ensayo, es necesario presentar las ideas de manera sistemática (Sánchez, 2001) y dicha sistematización es lo que se reconoce como organización retórica. Esta organización retórica se reflejó en la Tabla 4, que da razón de los patrones más comunes de organización dentro de las conclusiones en el ensayo de filosofía. Y tal como se ha visto, esto coincide con lo planteado por la literatura, que destaca los patrones $\mathrm{AB}, \mathrm{AD}$ y B. Ahora bien, es importante señalar, en relación con los patrones de organización retórica obtenidos en el análisis de los ensayos, la coincidencia con lo planteado por López et al. (2014) quienes, si bien no hablan de una organización retórica del ensayo propiamente tal, plantean las movidas más relevantes que deben darse en la conclusión de un ensayo, destacando la síntesis y el replanteamiento de ideas y argumentos, lo que es coherente con lo presentado en la Tabla 4.

En consecuencia, los hallazgos de este estudio señalan que existen 14 pasos retóricos que conforman la organización retórica de la movida conclusión de ensayos de filosofía. De estos pasos retóricos se destacan cuatro que se consideran prototípicos, a saber, identificación de la sección, incorporación de nuevos argumentos, replanteamiento de ideas y síntesis final.

En cuanto a la organización retórica del apartado, hay un patrón común que consiste en la identificación de la sección y síntesis final. Dicha identificación de la sección suele tener una presencia explícita, ya sea por medio de un subtítulo del tipo "conclusión" o subtítulos asociados como "palabras finales". Excepcionalmente, cuando el apartado no se encuentra identificado de forma explícita, este se identifica por medio de la presencia de los pasos llamados prototípicos.

En síntesis, las conclusiones de ensayos de filosofía centran la atención en el desarrollo de una síntesis final del tema tratado en el ensayo, lo que puede complementarse con la incorporación de nuevos argumentos y replanteamiento de ideas. La conclusión de los ensayos de filosofía, entonces, por medio de una síntesis final, en conjunto con los otros pasos retóricos prototípicos mencionados, permite reforzar la tesis del autor.

\section{Conclusiones}

Los pasos retóricos prototípicos que son parte de la estrategia del cierre discursivo de las conclusiones de ensayos de filosofía son: identificación de la sección, incorporación de nuevos argumentos, replanteamiento de ideas y síntesis final. Estos pasos retóricos, sobre todo los tres últimos mencionados, son los que permiten reforzar la tesis del autor en los ensayos de filosofía. 
Así como hay pasos retóricos prototípicos, se observan pasos retóricos que no son tan relevantes para la consecución de los propósitos comunicativos del género. Son los denominados pasos retóricos periféricos, o sea, mención de otras investigaciones, proyección, ejemplificación, preguntas retrospectivas y prospectivas, incorporación del lector, valoración del tema de tesis, cita final, cita de otra fuente y replanteamiento de tesis. Esto evidencia que en el cierre discursivo no es prioridad la interacción dialógica ni la valoración ni la proyección. Ahora bien, la ausencia de estos pasos retóricos periféricos en las conclusiones de ensayos de filosofía no perjudica el propósito del cierre discursivo, por lo que se pueden considerar pasos retóricos opcionales para dicho apartado.

Lo revisado en este estudio permite comprender la utilidad del análisis de género basándose en la observación de pasos retóricos, en este caso, de la movida conclusión de los ensayos que conformaron el corpus. Con dicho análisis se pudieron identificar los patrones de organización retórica de la conclusión, lo que finalmente permite entender que para los profesionales del área de la filosofía es necesario reafirmar la tesis planteada en el ensayo, por medio de una síntesis de ideas y argumentos.

Dada la relevancia de los resultados, obtenidos solo de la movida conclusión en ensayos de filosofía, sería interesante ampliar el análisis de los pasos retóricos y la descripción de la organización retórica a la totalidad del ensayo.

Se espera haber contribuido a la organización de este género y que ello sea de utilidad para su escritura en el discurso filosófico, así como también, que ofrezca bases para su enseñanza a estudiantes de la disciplina y de otras en las que el ensayo sea un género discursivo evaluativo. Finalmente, se confía en que estos hallazgos sean un aporte al campo de la lingüística aplicada y al estudio del discurso especializado, gracias a la descripción de los pasos retóricos, los propósitos comunicativos y la organización retórica de la conclusión de ensayos de filosofía.

\section{Referencias}

Angulo, N. (2013). El ensayo: algunos elementos para la reflexión. Innovación Educativa, 61(13), 107-121.

Arenas, M. (1997). Hacia una teoría general del ensayo: construcción del texto ensayístico (vol. 19). Ediciones de la Universidad de Castilla-La Mancha.

Boudon, E. \& Parodi, G. (2014). Artefactos multisemióticos y discurso académico de la economía: construcción de conocimientos en el género manual. Revista Signos, 47(85), 164-195.

Burdiles, G. (2016). Género caso clínico: organización retórica de su macromovida. Relato del caso en publicaciones médicas chilenas. Revista Signos, 49(91), 192-216. 
Calsamiglia, H. \& Tusón, A. (1999). Las cosas del decir: manual de análisis del discurso. Ariel.

Cerezo, P. (2002). El espíritu del ensayo. En F. García (ed.), El ensayo entre la filosofía y la literatura (pp. 1-30). Comares.

Ciapuscio, G. \& Otañi, I. (2002). Las conclusiones de los artículos de investigación desde una perspectiva contrastiva. Revista de Investigaciones Lingüísticas y Literarias Hispanoamericanas, 15, 117-133.

Espejo, C. (2006). La movida concluyendo en torno al tema de informes de investigación elaborados por estudiantes universitarios. Onomázein, 13(1), 35-54.

Farlora, M. (2015). Descripción funcional del género académico didáctico con función evaluativa prueba tipo ensayo: explorando el discurso de historia y psicología. En G. Parodi \& G. Burdiles (eds.), Leer y escribir en contextos académicos y profesionales: géneros, corpus y métodos (pp. 258-288). Ariel.

Figueroa, B. \& Aillón, M. (2015). Escritura académica de un ensayo mediado por el aprendizaje colaborativo virtual. Estudios Pedagógicos, 41(1), 79-91.

Fuentes, M. (2013). Las conclusiones de los artículos de investigación en historia. Literatura y Lingüística, 28, 215-232.

García-Ramírez et al. (2017). Validación del cuestionario de salud educativa. ReiDoCrea, 6, 64-73.

González, C. \& Burdiles, G. (2018). Organización retórica del género informe de estabilidad financiera: un contraste entre el informe del Banco Central de Chile y el Banco Federal Alemán. Círculo de Lingüística Aplicada a la Comunicación, $73,145-160$.

Hernández, R., Fernández, C. \& Baptista, P. (2010). Metodología de la investigación. McGraw Hill.

Hopkins, A. \& Dudley-Evans, T. (1988). A Genre-based Investigation of the Discussion Sections in Articles and Dissertations. English for Specific Purposes, 7, 113-12.

Ibáñez, R. \& Moncada F. (2017). El resumen en artículos de investigación científica. Spanish in Context, 14(2), 273-308.

Jonassen, D. \& Bosung, K. (2010). Arguing to Learn and Learning to Argue: Design Justifications and Guidelines. Educational Technology Research and Development, 58, 439-457.

Juárez, R. (2008). Escritura científica en ciencias de la salud. Revista Ateneo Argentino de Odontología, 47(1), 22-27. 
Julio, C. \& Parodi, G. (2017). El discurso profesional de la economía y de la filosofía: descripción genérico discursiva del Corpus PUCV-UCSC 2017. Presentación par SOCHIL, Universidad Católica del Maule.

López, M., Huerta, J., Ibarra, J. \& Almazán, K. (2014). Manual básico para la escritura de ensayos. Fundación SM de Ediciones México.

Olivares, M. \& González, P. (2014). Diagnóstico de las habilidades argumentativas orales de estudiantes de primer año de enfermería, kinesiología, nutrición y dietética y terapia ocupacional durante el segundo semestre de 2013. Revista Estudios Hemisféricos y Polares, 5(1), 104-120.

Oller, C. (2013). El ensayo argumentativo. En S. Solas et al. (Cord.), Introducción a la filosofía, argumentación filosófica, lectura académica (pp. 639-383). Editorial de la Universidad de La Plata.

Parodi, G. (2015). Géneros del discurso escrito: hacia una concepción integral desde una perspectiva sociocognitiva. En G. Parodi (ed.), Géneros académicos y géneros profesionales: accesos discursivos para saber y hacer (pp. 21-41). Ediciones Universitarias de Valparaíso.

Partarrieu, C. (2018). El ensayo argumentativo escrito por profesionales de la filosofía y por estudiantes de enseñanza media: una mirada a la sección conclusión (Tesis de pregrado). Universidad Católica de la Santísima Concepción, Concepción, Chile.

Rayas, L. \& Méndez, A. (2017). Los estudiantes universitarios ante la escritura del ensayo académico: dificultades y posibilidades. Innovación Educativa, 17(75), 123-148.

Reyes, A. (1959). Obras completas de Alfonso Reyes. Fondo de Cultura Económica.

Rodríguez, A. (2007). El ensayo académico: algunos apuntes para su estudio. Sapiens, $8(1), 147-159$.

Sabaj, 0., Toro, P. \& Fuentes, M. (2011). Construcción de un modelo de movidas retóricas para el análisis de artículos de investigación en español. Onomázein, $24(2), 245-271$.

Sánchez, C. (2001). Ensayos: anotaciones preliminares sobre su composición en el entorno escolar. Revista Educación y Pedagogía, 138(31), 135-148.

Schara, J. (1994). Comunicación, teoría, práctica y antología. Universidad del Valle de México.

Soler-Monreal, C. (2016). A Move-Step Analysis of the Concluding Chapters in Computer Science PhD Theses. Ibérica, 32, 105-132. 
Stagnaro, D. (2012). El artículo de investigación en economía: exploración de la estructura retórica de la conclusión. En V Congreso Internacional de Letras. 2697-2705.

Stagnaro, D. (2015). Configuraciones retórico-lingüísticas del resumen en artículos científicos de economía: contrastes en el interior de la disciplina. Revista Signos, 48(89), 425-444.

Swales, J. (1990). English in Academic and Research Settings. Cambridge University Press.

Swales, J. (2004). Research Genres: Explorations and Applications. Cambridge University Press.

Wingate, U. (2012). Argument! Helping Students Understand What Essay Writing Is About. Journal of English for Academic Purposes, (11), 145-154.

Zunino, C. \& Muraca, M. (2012). El ensayo académico. En L. Natale (coord.), En carrera: escritura y lectura de textos académicos y profesionales (pp. 61-77). Universidad Nacional de General Sarmiento. 